\title{
Analysis of Iron Meteorites Using Computed Tomography and Electron-probe Microanalysis
}

P. Carpenter*, D. C. Gillies**

* XD42 / BAE Systems / NASA, Marshall Space Flight Center, AL 35812, USA

** XD41 NASA, Marshall Space Flight Center, AL, 35812, USA

Computed tomography (CT) imaging and electron-probe microanalysis (EPMA) have been used to study samples of the Mundrabilla and Colomera iron meteorites in order to perform structural, textural, and mineralogical analysis [1]. Both $\gamma$-ray $\left({ }^{60} \mathrm{Co}\right.$ source, essentially monochromatic 1.25 $\mathrm{MeV}$ avg.) and $\mathrm{x}$-ray (420 KeV, continuous) sources have been used, with effective resolution of approximately $1 \mathrm{~mm}$ and $0.25 \mathrm{~mm}$, respectively. The $\gamma$-ray source provides $\sim 15 \mathrm{~cm}$ penetration through steel and is used for larger samples, whereas the $\mathrm{x}$-ray source provides superior resolution at reduced penetration but exhibits beam hardening artifacts. Here we present a combined approach where CT and EPMA imaging and microanalysis aid in the identification of structural and compositional features in iron meteorites.

CT imaging of Mundrabilla and Colomera provides $\sim 1 \mathrm{~mm}$ image slices which are animated as movies and used to render structural figures of the component phases (figures 1 and 2). The rendered CT data provide an excellent tool for visualizing and quantifying the structural relationships between component phases, and can be used for targeted selection of inclusions for sampling and further analysis. Analysis of the CT data indicates that large spatial features with significant density contrast are readily discriminated, whereas smaller features and phases of similar density are less effectively discriminated. In Mundrabilla we observe excellent discrimination between FeNi alloy (kamacite and taenite), FeS (troilite), and graphite-rich phases; however, due to density and resolution issues silicate inclusions have been difficult to identify. The CT data from Colomera are being used in combination with EPMA to refine the identification of silicate minerals. Colomera has silicate inclusions embedded in FeNi alloy that contain albitic glass, feldspar, and $\mathrm{Ca}-$ pyroxene (figures 3-5), and are clearly identified using CT and EPMA.

Conversion of raw CT data currently uses an ASTM method based on a library of elemental and compound standards, and it is anticipated that Monte Carlo modeling of $\gamma$-ray and x-ray absorption in simplified structures will aid in CT data reduction for iron meteorite samples. The analysis of rendered CT structures provides quantitative information about crystal growth and preliminary results indicate that Mundrabilla has a significant degree of FeNi connectivity, suggesting that dendritic growth occurred during directional solidification. Comparison to crystal growth experiments in other binary alloys may provide information about the solidification conditions [2]. Our goal is to better understand the complex crystallization and cooling histories of asteroid bodies as recorded by these iron meteorites.

We are honored to contribute to the extensive research performed on meteorites by Joe Goldstein. Assistance with the CT imaging was provided by Ron Beshears at MSFC and H. Pete Engel at Kennedy Space Center. Quantitative CT analysis was performed by Roberto Mendoza and Peter Voorhees at Northwestern University.

\section{References}

[1] D.C. Gillies et al., Microscopy and Microanalysis (2004), 720CD.

[2] R. Mendoza, et al., Microscopy and Microanalysis (2004), 74. 

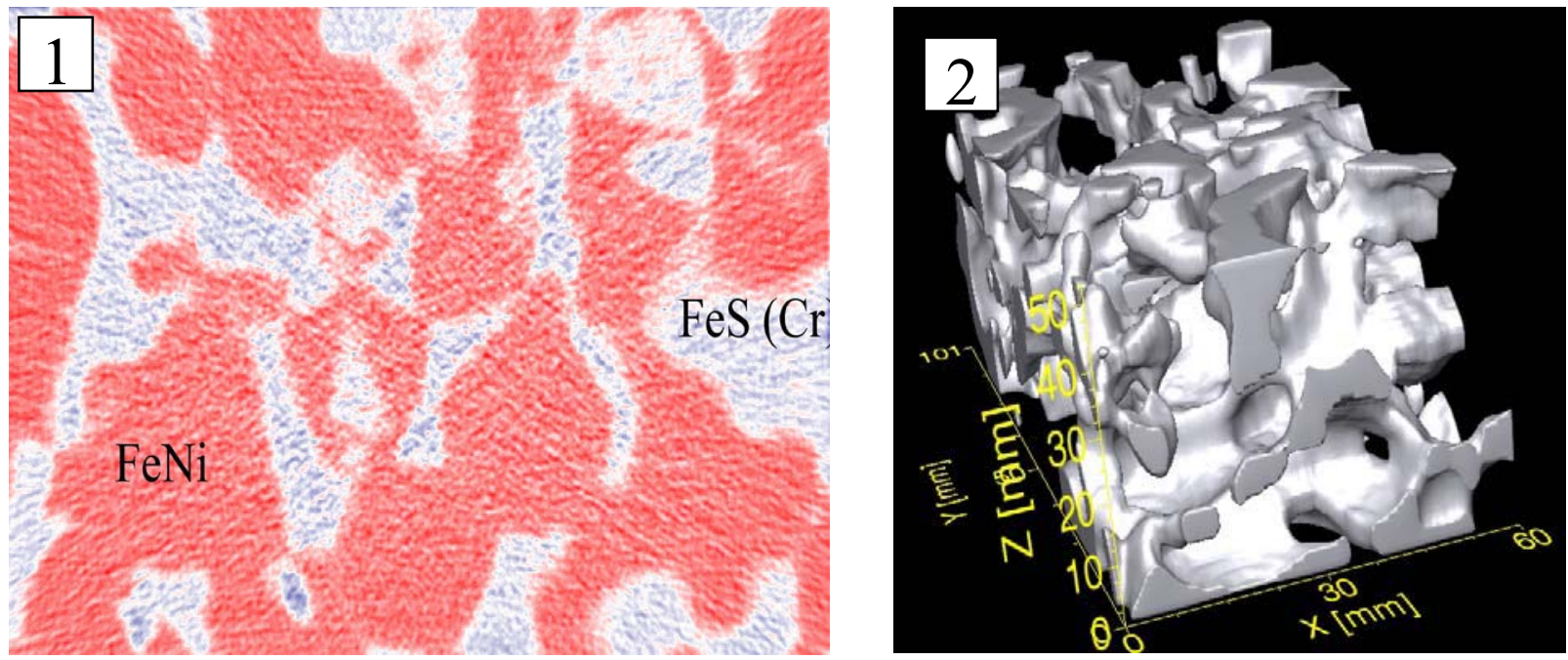

Fig. 1. CT image slice (cross section) of Mundrabilla sample. FeNi alloy is red, FeS is white. Sample width approximately $10 \mathrm{~cm}$.

Fig. 2. Rendered structure of FeS phase in Mundrabilla (FeNi alloy is transparent in this image).
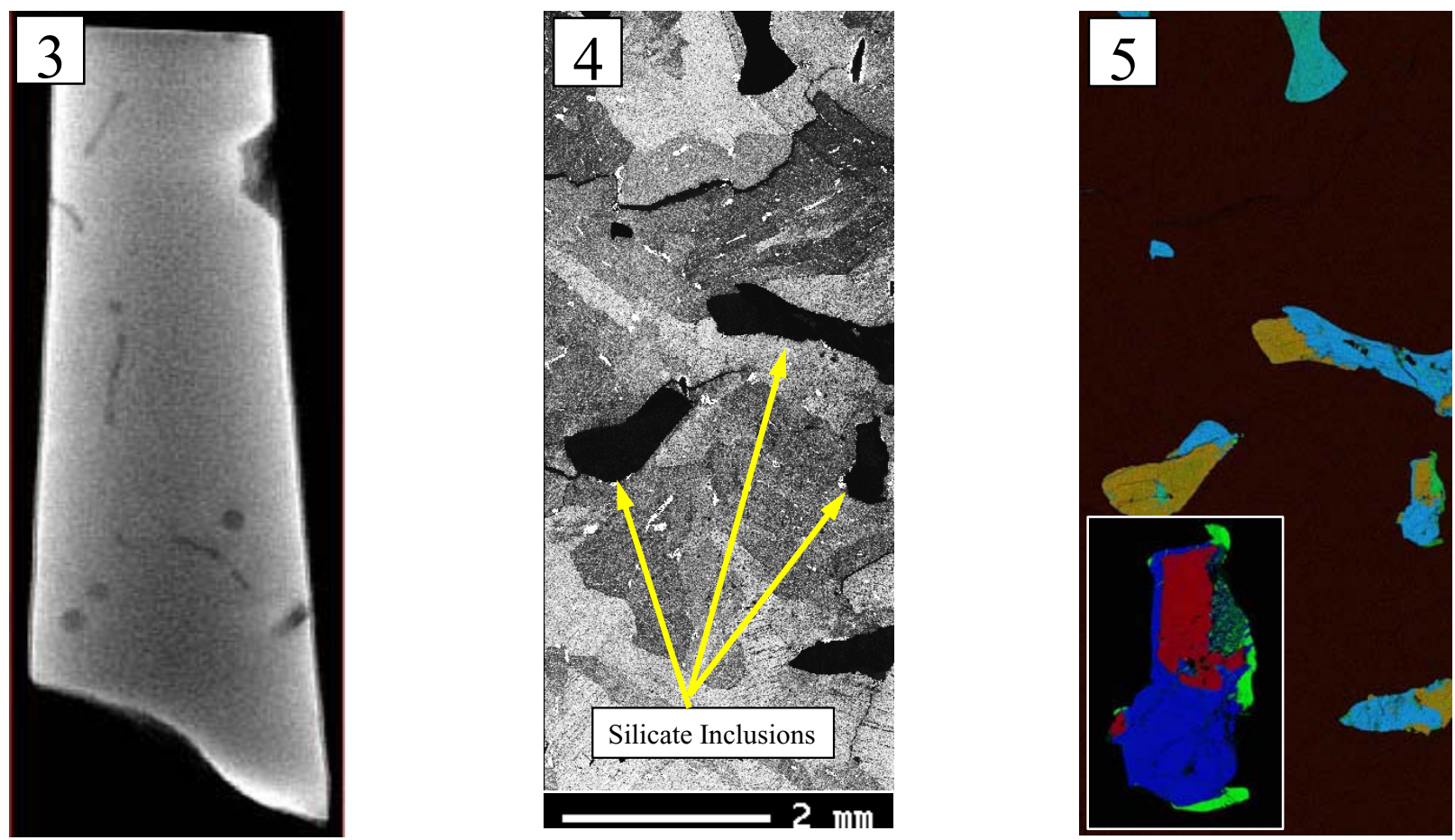

Fig. 3. CT longitudinal section of Colomera sample. Round and elongate inclusions (darker) contain albitic glass, feldspar, Ca-pyroxene, and accessory minerals. Sample length is approximately $10 \mathrm{~cm}$.

Fig. 4. Backscattered-electron stage-scan map of Colomera sample showing kamacite and taenite (FeNi alloy) with white schreibersite (FeNi phosphide) inclusions. Fig. 4 and 5 at same scale.

Fig. 5. Combination x-ray stage-scan map of Colomera showing silicate inclusions. Mineral colors are: Ca-pyroxene is gold, Na-rich feldspar and/or glass is blue, and K-rich feldspar is green. Inset shows inclusion at 3x higher magnification, with Ca-pyroxene in red, Na-rich feldspar in blue, and K-rich feldspar in green. 